\title{
Gene expression pattern in swine neutrophils after lipopolysaccharide exposure: a time course comparison
}

\author{
Gema Sanz-Santos', Ángeles Jiménez-Marín', Rocío Bautista², Noé Fernández², Gonzalo M Claros², Juan J Garrido ${ }^{*}$ \\ From International Symposium on Animal Genomics for Animal Health (AGAH 2010) \\ Paris, France. 31 May - 2 June 2010
}

\begin{abstract}
Background: Experimental exposure of swine neutrophils to bacterial lipopolysaccharide (LPS) represents a model to study the innate immune response during bacterial infection. Neutrophils can effectively limit the infection by secreting lipid mediators, antimicrobial molecules and a combination of reactive oxygen species (ROS) without new synthesis of proteins. However, it is known that neutrophils can modify the gene expression after LPS exposure. We performed microarray gene expression analysis in order to elucidate the less known transcriptional response of neutrophils during infection.
\end{abstract}

Methods: Blood samples were collected from four healthy Iberian pigs and neutrophils were isolated and incubated during 6, 9 and 18 hrs in presence or absence of lipopolysaccharide (LPS) from Salmonella enterica serovar Typhimurium. RNA was isolated and hybridized to Affymetrix Porcine GeneChip ${ }^{\circledR}$. Microarray data were normalized using Robust Microarray Analysis (RMA) and then, differential expression was obtained by an analysis of variance (ANOVA).

Results: ANOVA data analysis showed that the number of differentially expressed genes (DEG) after LPS treatment vary with time. The highest transcriptional response occurred at $9 \mathrm{hr}$ post LPS stimulation with 1494 DEG whereas at 6 and $18 \mathrm{hr}$ showed 125 and $108 \mathrm{DEG}$, respectively. Three different gene expression tendencies were observed: genes in cluster 1 showed a tendency toward up-regulation; cluster 2 genes showing a tendency for down-regulation at $9 \mathrm{hr}$; and cluster 3 genes were up-regulated at $9 \mathrm{hr}$ post LPS stimulation. Ingenuity Pathway Analysis revealed a delay of neutrophil apoptosis at $9 \mathrm{hr}$. Many genes controlling biological functions were altered with time including those controlling metabolism and cell organization, ubiquitination, adhesion, movement or inflammatory response.

Conclusions: LPS stimulation alters the transcriptional pattern in neutrophils and the present results show that the robust transcriptional potential of neutrophils under infection conditions, indicating that active regulation of gene expression plays a major role in the neutrophil-mediated- innate immune response.

\section{Background}

Neutrophils play a key role in innate immune response. They initiate phagocytosis, degranulation and killing without new synthesis of proteins. However, it has been demonstrated that new gene transcription and protein synthesis are required to maintain full capacity for

\footnotetext{
* Correspondence: ge1gapaj@uco.es

'Grupo de Genómica y Mejora Animal, Departamento de Genética, Facultad de Veterinaria, Universidad de Córdoba, Campus de Rabanales, Edificio Gregor Mendel C5, 14071 Córdoba, Spain

Full list of author information is available at the end of the article
}

human neutrophil phagocytosis and associated bactericidal activity $[1,2]$.

LPS treatment enhances neutrophil bactericidal activity, with an alteration in adhesion, respiratory burst, degranulation and motility $[3,4]$. Thus kinetic study of swine neutrophil response to LPS represents an in vitro model to investigate the innate immune response during bacterial infection.

To test the neutrophil transcriptional potential, global gene expression analysis was performed and the results indicated that the LPS-treated neutrophils increase their 
transcriptional activity by altering genes involved in different cellular processes including transcriptional regulation, cell signalling, cytoskeletal reorganization, etc. Furthermore, inhibition of apoptosis-related functions may indicate an increase of the neutrophil life span at 9 hr post LPS stimulation. Taken together, our results show that (1) relevant changes occurred in the pattern of gene expression of porcine neutrophils after LPS exposure, and (2) the biological functions identified to be meaningful using Ingenuity Pathway Analysis tool (IPA) suggests that these transcriptional changes play an important role in inflammation and immune response associated with LPS stimulation.

\section{Methods}

\section{Isolation and stimulation of neutrophils}

Blood samples were collected from 4 healthy Iberian pigs at the local abattoir. Neutrophils were isolated with dextran and ficoll sedimentation as previously described [5] and adapted for swine neutrophils. Isolated neutrophils were more than $99 \%$ pure and $<1 \%$ monocytes were present. Freshly purified neutrophils, $\sim 200 \times 10^{6}$ for each time point, were suspended at $5 \times 10^{6} \mathrm{ml}^{-1}$ in $40 \mathrm{ml}$ RPMI medium (Lonza, Basel, Switzerland) supplemented with $10 \%$ heat-inactivated bovine fetal serum. Stimulation was performed in $50 \mathrm{ml}$ Falcon tubes and rotated continuously at $37^{\circ} \mathrm{C}$ for 6,9 and $18 \mathrm{hr}$ in presence or absence of $100 \mathrm{ng} / \mathrm{ml}$ of LPS from $S$. Typhimurium. Neutrophil activation was checked by measuring the released IL- 8 in the culture medium by ELISA. The manufacturer certified all reagents (serum, buffers, media and containers) as nonpyrogenic.

\section{RNA purification and microarray hybridizations}

RNA was isolated with RNeasy columns (Qiagen, Valencia, CA) based on the manufacturer's protocol. Eluted RNA was treated with RNase-Free DNase Set (Qiagen, Valencia, CA) and the integrity, quality and quantity of RNA were checked in the Agilent Bioanalyser 2100 (Agilent Technologies, Palo Alto, CA) and NanoDrop1000 Spectrophotometer (Thermo Scientific, Wilmington, DE).

Biotinylated cRNA was obtained following the TwoCycle Eukaryotic Target Labeling System from Affymetrix. The labeled cRNA is then cleaned up, fragmented, and hybridized to Affymetrix Porcine GeneChip according to the manufacturer's procedures (Expression Analysis Technical Manual, Affymetrix Inc., Santa Clara, CA). Chips were scanned with an Affymetrix GeneChip Scanner 3000 (Affymetrix Inc., Santa Clara, CA).

\section{Data analysis}

All data were normalized using the affy library of the Bioconductor package (Gentleman et al., 2004) for R
(2.10) and adapted in-house scripts. Features appearing in all replicates and having a signal of 50 over background were normalized using robust multi-array analysis (RMA) [6]. Genes were filtered for based on Affymetrix's present/marginal/absent (PMA) calls using mismatch probe intensity, the ratio of PM to MM, only those probes present in at least $80 \%$ of the slide was used in the analysis of microarray. Differential expression and clustering was performed with Spotfire DecisionSite v 9.0 (SP2) as follows: feature values were obtained by a one-way analysis of variance (ANOVA) with a $p$-value $<0.05$ and hierarchically clustered using UPGMA method and Pearson's correlation as the similarity measure. Since the Affymetrix Porcine GeneChip ${ }^{\circledR}$ is not fully annotated in all the features, it was re-annotated with Blast2GO [7] with a minimum E-value of 1010 and a minimum similarity of $50 \%$.

\section{Biological interpretation}

The networks, functional analysis and canonical pathways were generated through the use of Ingenuity Pathway Analysis (Ingenuity Systems ${ }^{\circledR}$, http://www.ingenuity. com). IPA provides computational algorithms to identify and generate significant biological networks and pathways that are particularly enriched with our genes of interest. Networks are ranked by a score that takes into account the number of focus genes and the size of the networks, indicating the likelihood of the focus genes in a network being found together by chance. The higher the score $($ score $=-\log (\mathrm{p}$-value $))$, the lower the probability of finding the observed Network Eligible Molecules in a given network by chance. IPA also gives information on biological functions and canonical pathways. Thus, DEG were grouped into known biological functions, networks and canonical pathways based on human and rodent studies.

\section{Results and discussion}

\section{Changes in gene expression profile in neutrophils stimulated with LPS}

In this study, we performed microarray analysis in order to elucidate the response of the porcine neutrophils to LPS from $S$. Typhimuriun along a time course of 6, 9 and $18 \mathrm{hr}$ in vitro stimulation. To achieve that, ANOVA adapted for time course experiments was used, in order to identify genes whose temporal expression patterns differ between control and LPS-treated samples. As a result, 1090 genes were differentially expressed along the time course and the resulting hierarchical clustering is shown in Figure 1. Moreover, genes were clustered according to idealized expression patterns. The query gene is assigned to a cluster designated by the idealized pattern that has the maximal correlation with that gene. Figure 2 shows the three gene expression clusters 


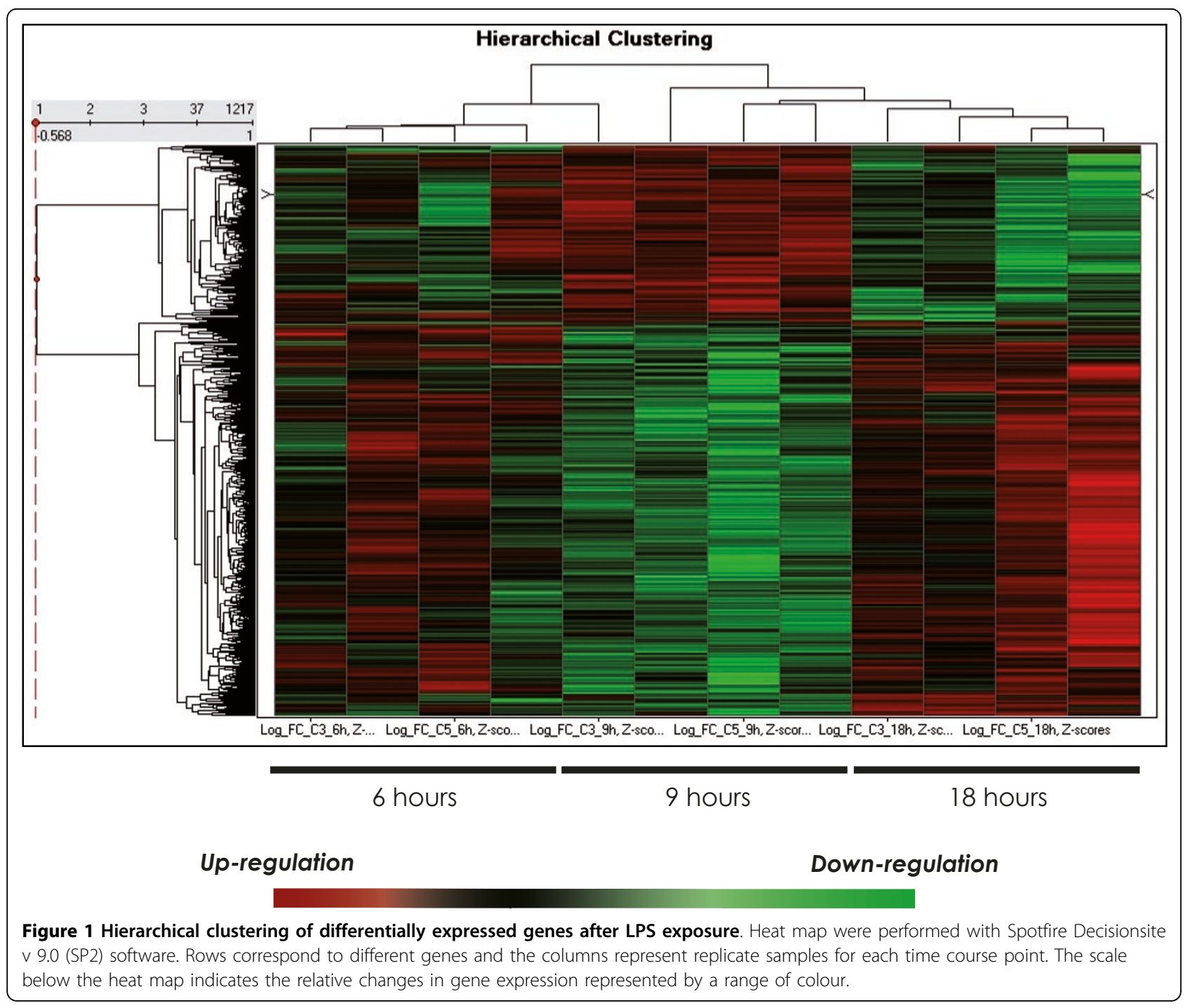

obtained in this analysis. This criterion for classifying genes according to their similarity to an idealized expression pattern allows us to determine the gene expression tendency through the time course. Clustering results reveal distinct temporal patterns and the most significant transcriptional changes happened after $9 \mathrm{hr}$ post LPS exposure. Individual ANOVA tests for each time point confirm this finding as shown in Figure 3. Thus, at 6 and $18 \mathrm{hr}$, there are 125 and 108 DEG, respectively, whereas at $9 \mathrm{hr}$, there are 1494 DEG.

\section{Biological analysis with Ingenuity Pathway Analysis}

IPA identified significant networks, top functions and canonical pathways associated with the DGE for the time course analysis. Networks with score higher than 25 (except for cluster 1) are presented in Additional file 1. Focus genes are shown in bold. Top biofunctions and canonical pathways are listed in Additional file 2.
Cluster 1 contains 8 genes with tendency for up-regulation through the time course, including genes coding for products involved in metabolism (GRHPR, SLC38A1) and immune-related functions, such as adhesion, cellular movement and phagocytosis (SIRPA, SMARCA4) or linked to glucocorticoid signaling (HSPA14), which is known to be implicated in inflammatory response and apoptosis [8].

Cluster 2 includes 747 down-regulated genes at $9 \mathrm{hr}$. These molecules are involved in cellular organization, molecular transport and protein trafficking and are related to canonical pathways such as JAK/STAT signaling, Cdc42 signaling and protein ubiquitination. Network 3 (Additional file 3) is related to molecular transport, protein trafficking and cellular development. This network is focused on TGFB, a cytokine implicated in process such as neutrophil locomotion, communication among immune cells or glucocorticoid receptor signaling [9]. 


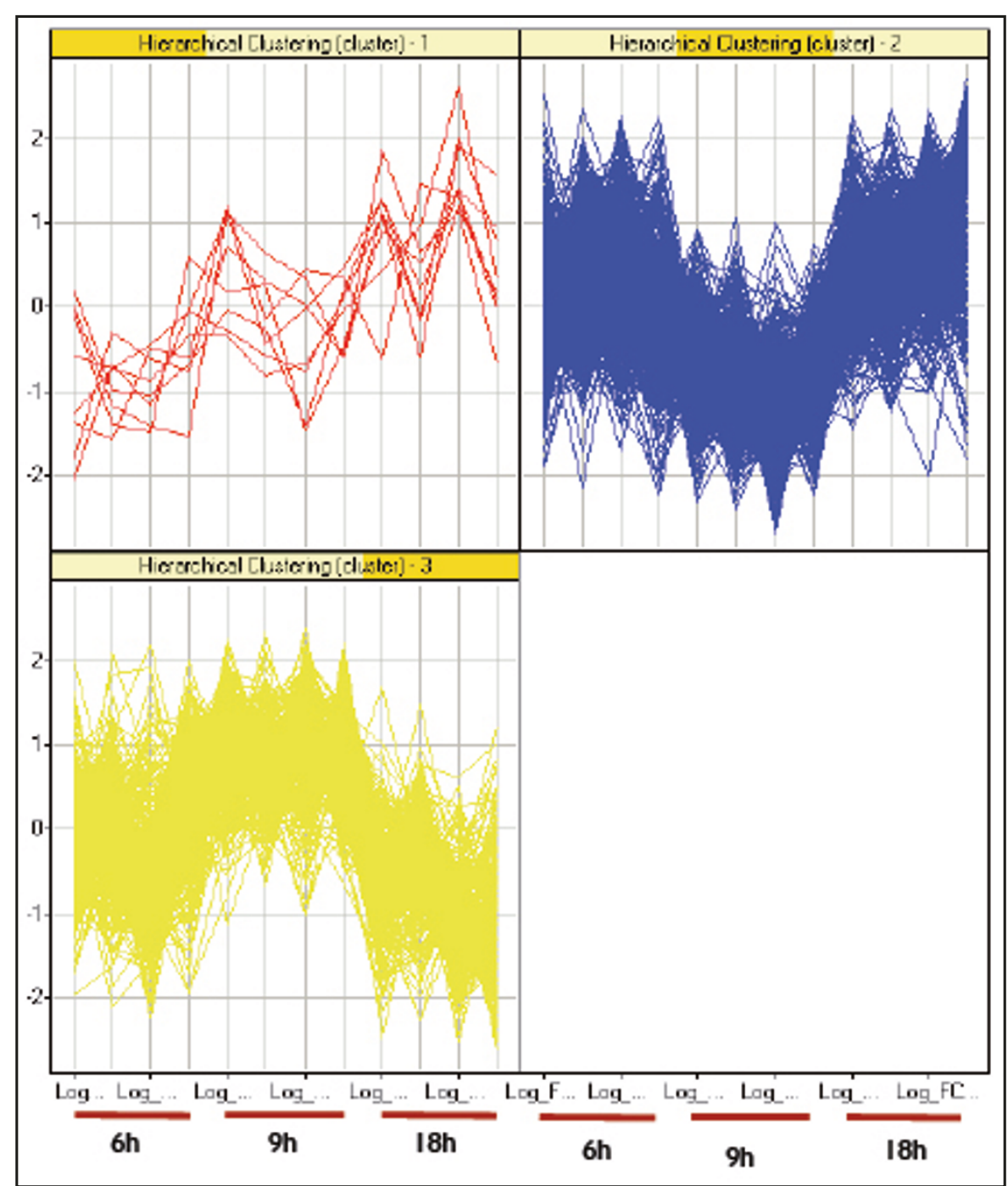

Figure 2 Differentially expressed genes grouped into three different clusters. Cluster 1 contains 8 genes with up-regulation tendency through the time course. 747 genes belonging the cluster 2, with a down-regulation tendency at 9 hr. Opposite tendency can be observed in the cluster 3, where 335 genes show an up-regulation at $9 \mathrm{hr}$ and down-regulation at $18 \mathrm{hr}$.

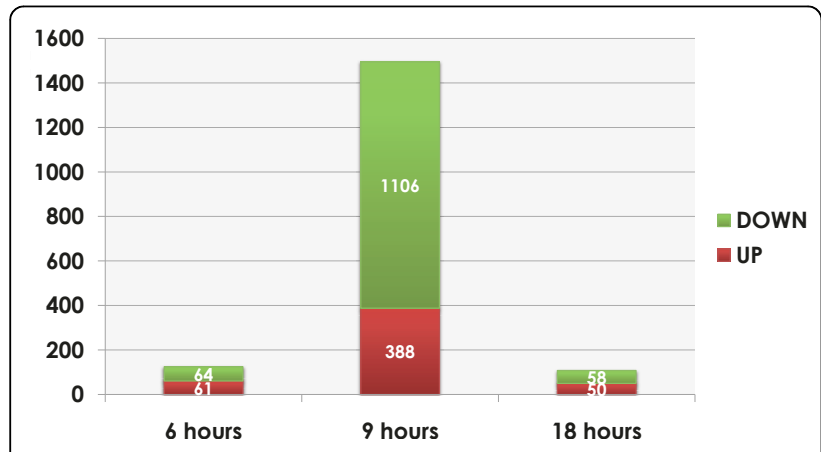

Figure 3 Differentially expressed genes in each time point. 125 and 108 genes were altered at 6 and 18 hr respectively, with a similar number of up and down-regulated genes. Most significant transcriptional changes were observed at $9 \mathrm{hr}$ post LPS stimulation. 1106 genes were down-regulated and 388 were up-regulated.
Finally, cluster 3 consists of 335 up-regulated genes. Functions associated with these molecules are related to cellular assembly and reorganization, cellular maintenance and gene expression. Canonical pathways are related to protein ubiquitination signaling, PDGF signaling and IL-3 signaling which is involved in cell survival by activation of JAK/STAT signaling and BCL2 [10]. Network 2 (Additional file 4) highlights NF- $\kappa \mathrm{B}$ interactions and covers several canonical pathways such as acute phase response signaling and interferon signaling.

\section{Inhibition of spontaneous apoptosis at 9 hrs}

Turnover of aging neutrophils occurs in the absence of activation through a process known as spontaneous 
apoptosis [11] and this ability of aged neutrophils to undergo apoptosis plays a central role in resolution of the acute inflammatory response. A well-described effect of LPS is its ability to inhibit spontaneous neutrophil apoptosis $[12,13]$. Thus, a delay of the apoptosis function could be related to the down-regulation of apoptosis mediators such as CASP6, ROCK1, CFLAR and DIABLO (Figure 4) shown at $9 \mathrm{hr}$ post stimulation. In addition, BCL2, an anti-apoptotic member of the Bcl-2 family of apoptosis regulator proteins [14] was up-regulated at $9 \mathrm{hr}$.

MCL1 encodes myeloid leukemia cell differentiation protein, Mcl-1, that also belongs to the Bcl-2 family.
Alternative splicing occurs at this locus and two transcript variants have been identified. The longer gene product (isoform 1) enhances cell survival whereas the shorter gene product (isoform 2) promotes apoptosis [15]. MCL1 is down-regulated at $9 \mathrm{hr}$ suggesting that it could be the shorter isoform of the Mcl-1 protein.

Also, there is a down-regulation of transcripts coding for some anti-bacterial products like NCF1, NCF2 and NCF4. The reactive oxygen system is autocytotoxic for neutrophils and its down-regulation may contribute to the prolongation of life span during inflammation. Therefore, many components of cell survival pathways are regulated by LPS, and these gene products may be

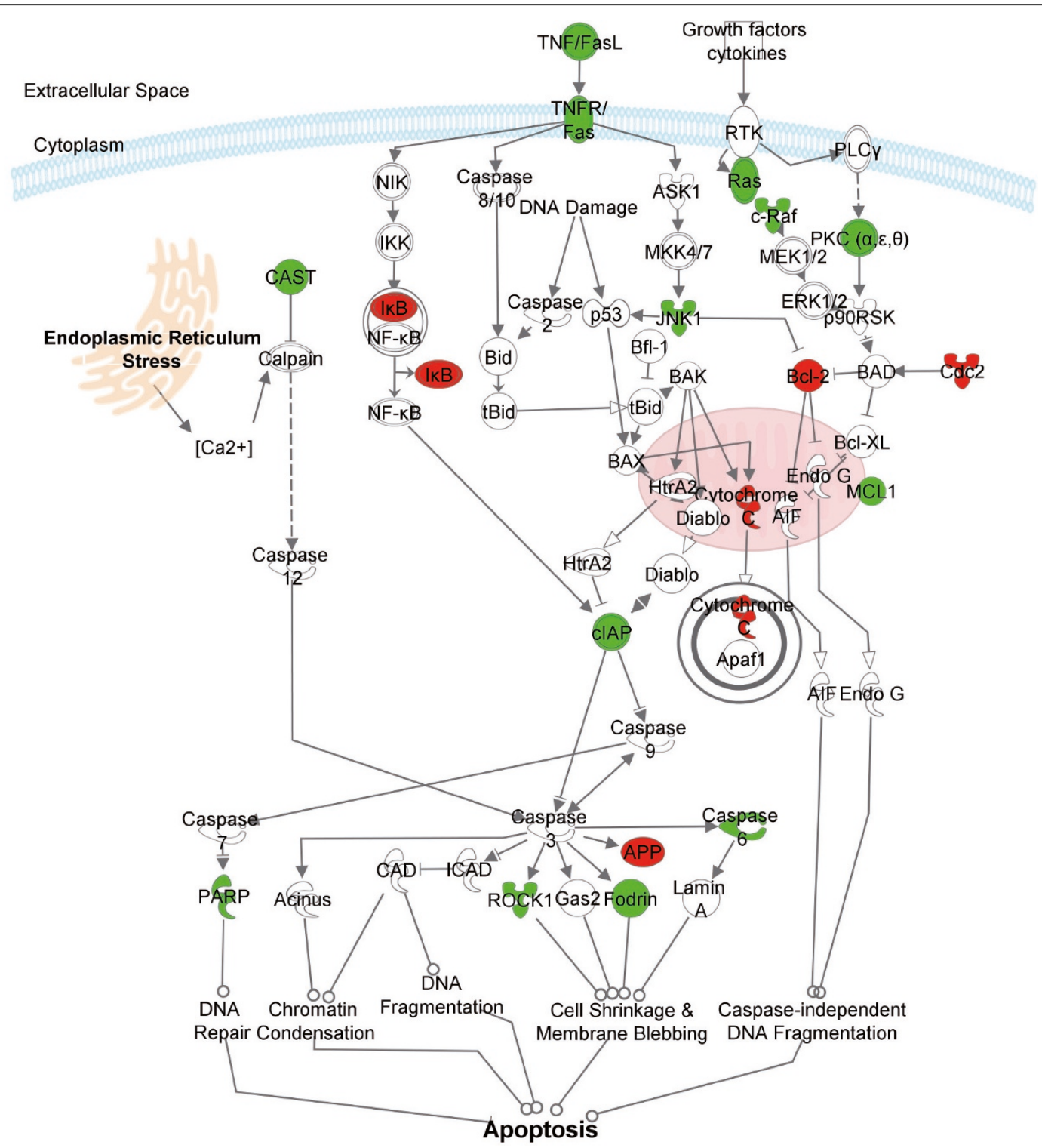

Figure 4 Delay in apoptosis at $\mathbf{9} \mathbf{~ h r}$. Canonical pathway obtained with IPA for apoptosis signaling. Up and Down -regulated genes are in red and green, respectively. 
involved in maintaining neutrophil viability. Inhibition of neutrophil apoptosis may improve the host defense against infection by increasing functional longevity of the cells at the inflammatory sites. The results of this study indicate that activated neutrophils not only generate cytokines or phagocytic-related response but also changes in the expression of genes coding for receptors, transcription factors, chromatin remodeling, cytoskeleton reorganization and apoptosis-related genes.

\section{Additional material}

Additional file 1: IPA Networks Networks are selected if their scores are higher than 25 for each cluster (except for cluster 1, because the number of genes is too small to analyze with IPA). The table contains columns with the network number, the name of the comparison list, names of the genes involved in the network, the score value and the top functions. Focus genes are shown in bold.

Additional file 2: IPA Biofunctions and Canonical Pathways Top 10 IPA biofunctions and canonical pathways. a) Biofunctions: Tested by the Fisher Exact test $p$-value. b) Canonical Pathway. For each list, the pathways are ranked by the score [score $=-\log (p$-value $)]$ using the same criterions than biofunctions. The table includes the ratio (number of focus molecules in a given pathway divided by the total number of the molecules that conform that pathway)

Additional file 3: Network $\mathbf{3}$ from gene cluster $\mathbf{2}$ The top functions of this network are Molecular transport, protein trafficking and cellular development. This network is focused on TGFB1 molecule (orange circle). Canonical pathways and functions are overlaid with red and yellow edges, respectively.

Additional file 4: Network 2 from gene cluster 3 The top functions of this network are Infection mechanism, gene expression, and cell death. This network is focused on NF- $\kappa$ B molecule (purple circle). Canonical pathways and functions are overlaid with red and yellow edges, respectively.

\footnotetext{
Acknowledgements

Authors acknowledge the computer resources and the technical support provided by the Plataforma Andaluza de Bioinformatica of the University of Malaga. This work was supported by EU funds provided by EADGENE project, and by the project of the Spanish Ministry of Science and Innovation AGL2008-0400.

This article has been published as part of BMC Proceedings Volume 5 Supplement 4, 2011: Proceedings of the International Symposium on Animal Genomics for Animal Health (AGAH 2010). The full contents of the supplement are available online at http://www.biomedcentral.com/1753$6561 / 5$ ? issue $=$ S4
}

\section{Author details}

'Grupo de Genómica y Mejora Animal, Departamento de Genética, Facultad de Veterinaria, Universidad de Córdoba, Campus de Rabanales, Edificio Gregor Mendel C5, 14071 Córdoba, Spain. ²Plataforma Andaluza de Bioinformática, Universidad de Málaga, Edificio de Bioinnovación, C/Severo Ochoa 34, Parque Tecnológico de Andalucía, 29590 Málaga, Spain.

\section{Authors' contributions}

GS carried out the experimental and IPA work with AJM. RB, NF and GC conducted the statistical analysis of microarray data. JJG conceived, coordinated and supervised the research. GSS drafted the manuscript which was approved by all the authors.

\section{Competing interests}

The authors declare that they have no competing interests.
Published: 3 June 2011

\section{References}

1. Homburg CH, Ross D: Apoptosis of neutrophils. Curr Opin Hematol 1996, 3:94-99.

2. Kasprisin DO, Harris MB: The role of RNA metabolism in polymorphonuclear leukocyte phagocytosis. J Lab Clin Med 1977, 90:118-124.

3. Cohn ZA, Morse SI: Functional and metabolic properties of polymorphonuclear leucocytes II. The influence of a lipopolysaccharide endotoxin. J Exp Med 1960, 111:689-704.

4. Dahinden CA, Galanos C, Fehr J: Granulocyte activation by endotoxin I. Correlation between adherence and other granulocyte functions, and role of endotoxin structure on biologic activity. J immunol 1983, 130:857-862.

5. Heit B, Liu L, Colarusso P, Puri KD, Kubes P: PI3K accelerates, but is not required for, neutrophil chemotaxis to fMLP. J Cell Sci. 2008, 121(Pt 2):205-14.

6. Irizarry RA, Hobbs B, Collin F, Beazer-Barclay YD, Antonellis KJ, Scherf U, Speed TP: Exploration, normalization, and summaries of high density oligonucleotide array probe level data. Biostatistics 2003, 4(2):249-64.

7. Conesa A, Götz S, García-Gómez JM, Terol J, Talón M, Robles M: Blast2GO: a universal tool for annotation, visualization and analysis in functional genomics research. Bioinformatics 2005, 21(18):3674-3676.

8. Franchimont D: Overview of the actions of glucocorticoids on the immune response: a good model to characterize new pathways of immunosuppression for new treatment strategies. Ann N Y Acad Sci 2004 1024:124-37.

9. Taylor AW: Review of the activation of TGF- $\beta$ in immunity. J Leukoc Biol 2009, 85:29-33.

10. Reddy EP, Korapati A, Chaturvedi P, Rane S: IL-3 signalling and the role of Src kinases, JAKs and STATs: a covert liaison unveiled. Oncogene 2000, 19(21):2532-47.

11. Savill J: Apoptosis in resolution of inflammation. J Leukoc Biol 1997, 61:375-380.

12. DeLeo FR: Modulation of phagocyte apoptosis by bacterial pathogens. Apoptosis 2004, 9:399-413.

13. Lee A, Whyte MK, Haslett C: Inhibition of apoptosis and prolongation of neutrophil functional longevity by inflammatory mediators. J Leukoc Biol 1993, 54:283-288.

14. Hockenbery D, Nunez G, Milliman C, Schreiber RD, Korsmeyer SJ: BCl-2 is an inner mitochondrial membrane protein that blocks programmed cell death. Nature 1990, 348:334-336.

15. Bae J, Leo CP, Hsu SY, Hsueh AJW: MCL-1S, a splicing variant of the antiapoptotic $B C L-2$ family member $\mathrm{MCL}-1$, encodes a proapoptotic protein possessing only the $\mathrm{BH} 3$ domain. J. Biol. Chem 2000, 275:25255-25261.

doi:10.1186/1753-6561-5-S4-S11

Cite this article as: Sanz-Santos et al:: Gene expression pattern in swine neutrophils after lipopolysaccharide exposure: a time course comparison. BMC Proceedings 2011 5(Suppl 4):S11.

\section{Submit your next manuscript to BioMed Central and take full advantage of:}

- Convenient online submission

- Thorough peer review

- No space constraints or color figure charges

- Immediate publication on acceptance

- Inclusion in PubMed, CAS, Scopus and Google Scholar

- Research which is freely available for redistribution 